Pacific Journal of Mathematics

HIGHLY PROXIMAL AND GENERALIZED ALMOST FINITE 


\title{
HIGHLY PROXIMAL AND \\ GENERALIZED ALMOST FINITE EXTENSIONS OF MINIMAL SETS
}

\author{
Peter S. Shoenfeld
}

\begin{abstract}
Highly proximal extensions are a nonmetric generalization of the notion of almost one-to-one extensions of minimal flows. These extensions are studied and the results are applied to the Veech structure theorem and to generalized almost finite homomorphisms.
\end{abstract}

1. Introduction. In Veech's paper on point distal flows [7], hyperspaces are used to associate an almost one-to-one homomorphism with a given homomorphism of metric minimal sets. In $\S 2$, the notion of a highly proximal homomorphism is used to obtain a nonmetric generalization of this construction. An abstract characterization is obtained and a strengthened version of the Veech Structure Theorem is proved. Generalized almost finite homomorphisms are studied in $\S 3$.

A flow $(X, T)$ consists of a discrete group, $T$, acting on a compact Hausdorff space $X$ as a group of homeomorphisms. Since $T$ remains fixed in this paper, we will write $X$ instead of $(X, T)$. An extension, or homomorphism, is a continuous, equivariant map. Given a family of homomorphisms $\left\{\psi_{i}: X_{i} \rightarrow Y\right\}$, its product is the homomorphism $\psi: X \rightarrow Y$ where $X=\left\{\left\langle x_{i}\right\rangle \in \Pi_{i}\left\{X_{i}\right\} \mid \psi_{i}\left(x_{i}\right)\right.$ the same for all $\left.i\right\}$, with the group action and $\psi$ defined in the obvious way. We assume the basic material in [3] concerning $\beta T$, almost periodicity, universal minimal sets, etc. We distinguish a universal minimal set $M$ and let $J=$ idempotents in $M\}$. Given $x \in X$, we define $J(x)=\{u \in J \mid x u=x\}$. We will generally deal with a fixed homomorphism $\pi: X \rightarrow Y$, with $X$ and $Y$ minimal.

The hyperspace flow $2^{X}$ is the space of all closed, nonempty subsets of $X$ with the Hausdorff topology and with the group action defined in the obvious way. We will use the subflow $2^{\pi}=\left\{A \in 2^{X} \mid A \subset \pi^{-1}(y)\right.$ for some $y \in Y\}$ and the naturally defined homomorphism $\bar{\pi}: 2^{\pi} \rightarrow Y$. Given $A \in 2^{x}, p \in \beta T$, we let $A \circ p$ denote the action of $p$ on $A$ within $2^{x}$ and let $A p=\{x p \mid x \in A\}$. Generally $A \circ p \neq A p$. It is easily seen that $x \in A \circ p$ if and only if $x_{n} t_{n} \rightarrow x$ for some nets $\left\langle x_{n}\right\rangle$ in $A$ and $\left\langle t_{n}\right\rangle$ in $T$ such that $t_{n} \rightarrow p$. We may define $A \circ p$ this way when the set $A \subset X$ is not necessarily closed. It is then readily shown that $A \circ p=\bar{A} \circ p$. 
Basic material on hyperspace flows may be found in [5] and [6]. The following lemma from [6] will be used frequently.

LEMMA 1.1. The following statements hold for any homomorphism $\pi: X \rightarrow Y$, without minimality assumptions.

(i) $\pi(A) \circ p=\pi(A \circ p)$ for all $A \in 2^{X}, p \in \beta T$.

(ii) $\pi^{-1}(B) \circ p \subset \pi^{-1}(B \circ p)$ for all $B \in 2^{Y}, p \in \beta T$.

(iii) $\pi^{-1}(y) \circ p \subset \pi^{-1}(y p)$ for all $y \in Y, p \in \beta T$.

(iv) $\pi^{-1}(y) \circ(p q)=\pi^{-1}(y p) \circ q$ for all almost periodic points $y \in Y$ and $p, q \in M$.

Next we use hyperspaces to get a new characterization of almost periodic homomorphisms. The following lemma is well known.

LEMma 1.2. Suppose $S$ is a dense subset of the equivalence relation determined by $\pi$ and $x, x^{\prime}, x^{\prime \prime} \in X$. Then the pair $\left(x, x^{\prime}\right)$ is regionally proximal relative to $\pi$ iff there exist nets $\left\langle x_{n}\right\rangle,\left\langle x_{n}^{\prime}\right\rangle$ in $X$, and $\left\langle t_{n}\right\rangle$ in $T$ such that each $\left(x_{n}, x_{n}^{\prime}\right) \in S$ and

$$
\begin{array}{ll}
x_{n} \rightarrow x & x_{n} t_{n} \rightarrow x^{\prime \prime} \\
x_{n}^{\prime} \rightarrow x^{\prime} & x_{n}^{\prime} t_{n} \rightarrow x^{\prime \prime} .
\end{array}
$$

Lemma 1.3. Suppose $\bar{\pi}$ is distal. Consider $x, x^{\prime} \in X, y \in Y, p \in$ $\beta T$, and nets $\left\langle x_{n}\right\rangle$ in $\pi^{-1}(y)$ and $\left\langle t_{n}\right\rangle$ in $T$ such that $x_{n} \rightarrow x, t_{n} \rightarrow p$, and $x_{n} t_{n} \rightarrow x^{\prime}$. Then $x^{\prime}=x p$.

Proof. Let $A_{n}=\left\{x_{n^{\prime}} \mid n^{\prime} \geqq n\right\}$ for each $n$. Pick $u \in J(y)$. Then $A \circ u=A$ for each closed $A \subset \pi^{-1}(y)$, since $\bar{\pi}$ is distal, and, in particular, $A_{n} \circ u=\bar{A}_{n} \circ u=\bar{A}_{n}$. Also $x u=x$. Clearly, $\cap_{n}\left\{\bar{A}_{n}\right\}=\{x\} \quad$ and $x^{\prime} \in \cap_{n}\left\{\bar{A}_{n} \circ p\right\}$. Pick $q \in M$ so $q=q u$ and $u p q=q p u=u$. Now

$$
\begin{aligned}
\{x\} & =\cap_{n}\left\{\bar{A}_{n}\right\}=\cap_{n}\left\{\overline{A_{n}} \circ(u p q)\right\} \\
& =\cap_{n}\left\{\left(\bar{A}_{n} \circ p\right) \circ q\right\} \supset\left(\cap_{n}\left\{\bar{A}_{n} \circ p\right\}\right) \propto \supset\left\{x^{\prime} q\right\}
\end{aligned}
$$

and so $x=x^{\prime} q . \quad$ Pick $v \in J$ such that $u p v=u p . \quad$ Let $y^{\prime}=\pi\left(x^{\prime}\right)$. Then $y^{\prime}=\pi\left(x^{\prime}\right)=\pi\left(\lim x_{n} t_{n}\right)=\lim \left(\pi\left(x_{n} t_{n}\right)\right)=\lim y t_{n}=y p=y u p=y u p v=y^{\prime} v$. We have $x^{\prime}=x^{\prime} v$ also, since $\bar{\pi}$ is distal. Finally, $x^{\prime}=x^{\prime} v=x^{\prime} u v=$ $x^{\prime} q p u v=x p u v=x u p v=x u p=x p$.

THEOREM 1.4. The following are equivalent:

(i) $\pi$ is almost periodic.

(ii) Each element of $2^{\pi}$ is almost periodic.

(iii) $\bar{\pi}$ is distal.

(iv) $\bar{\pi}$ is almost periodic. 
Proof. (iv) $\Rightarrow$ (iii) $\Rightarrow$ (ii) is clear.

(i) $\Rightarrow$ (iv). Suppose $A, A^{\prime}$ are relatively regionally proximal (relative to $\bar{\pi})$ in $2^{\pi}$. Then there exists $B \in 2^{\pi}$, nets $\left\langle A_{n}\right\rangle,\left\langle A_{n}^{\prime}\right\rangle$ in $2^{\pi},\left\langle y_{n}\right\rangle$ in $Y$, and $\left\langle t_{n}\right\rangle$ in $T$ such that $\bar{\pi}\left(A_{n}^{\prime}\right)=y_{n}$ for each $n$ and

$$
\begin{array}{ll}
A_{n} \rightarrow A & A_{n} t_{n} \rightarrow B \\
A_{n}^{\prime} \rightarrow A^{\prime} & A_{n}^{\prime} t_{n} \rightarrow B .
\end{array}
$$

We must show $A=A^{\prime}$. Consider $x \in A$; it will suffice to show $x \in A^{\prime}$. Taking subnets, there exists $x_{n} \in A_{n}$, for each $n$, such that $x_{n} \rightarrow x$. Taking a subnet again, $x_{n} t_{n} \rightarrow x_{0}$ for some $x_{0} \in B$. Taking subnets two more times, we can find $x_{n}^{\prime} \in A_{n}^{\prime}$ such that $x_{n}^{\prime} t_{n} \rightarrow x_{0}$ and $x^{\prime} \in A^{\prime}$ such that $x_{n}^{\prime} \rightarrow x^{\prime}$. We now have $\pi\left(x_{n}\right)=\pi\left(x_{n}^{\prime}\right)=y_{n}$, for each $n$, and

$$
\begin{array}{ll}
x_{n} \rightarrow x & x_{n} t_{n} \rightarrow x_{0} \\
x_{n}^{\prime} \rightarrow x^{\prime} & x_{n}^{\prime} t_{n} \rightarrow x_{0} .
\end{array}
$$

Since $\pi$ is assumed almost periodic, we have $x=x^{\prime}$ so $x \in A^{\prime}$ as required.

(ii) $\Rightarrow$ (iii). Consider $y \in Y, \quad u \in J(y)$. Given $A \in 2^{\pi}$ with $\bar{\pi}(A)=y$, it will suffice to show that $A \circ u=A$. Clearly, the elementwise almost periodicity of $2^{\pi}$ implies that $\pi$ is distal so that $B w=B$ whenever $B \subset \pi^{-1}(y)$ and $w \in J(y)$. Thus $A=A u \subset A \circ u$. Since $2^{\pi}$ is element-wise almost periodic, we have $A \circ v=A$ for some $v \in J(y)$. Therefore $A \circ u=(A \circ u) v \subset(A \circ u) \circ v=A \circ(u v)=A \circ v=A$.

(iii) $\Rightarrow$ (i). Suppose $x, x^{\prime}$ relatively regionally proximal, with $\pi(x)=\pi\left(x^{\prime}\right)=y$. We must show $x=x^{\prime}$. Now $\pi$ is distal since $\bar{\pi}$ is. Therefore $\pi$ is an open map. Thus, for every $y^{\prime} \in Y$, there exists a net $\left\langle t_{n}\right\rangle$ in $T$ such that $\pi^{-1}(y) t_{n} \rightarrow \pi^{-1}\left(y^{\prime}\right)$. Hence $\left(\pi^{-1}(y) \times \pi^{-1}(y)\right) T$ is dense in the equivalence relation determined by $\pi$. By Lemma 1.2 there exist nets $\left\langle x_{n}\right\rangle,\left\langle x_{n}^{\prime}\right\rangle$ in $\pi^{-1}(y)$ and $\left\langle t_{n}\right\rangle,\left\langle s_{n}\right\rangle$ in $T$ such that
(a) $x_{n} t_{n} \rightarrow x$
(b) $x_{n} t_{n} s_{n} \rightarrow x$
(c) $x_{n}^{\prime} t_{n} \rightarrow x^{\prime}$
(d) $x_{n}^{\prime} t_{n} s_{n} \rightarrow x$.

Taking subnets, we can find $x_{1}, x_{1}^{\prime} \in \pi^{-1}(y)$ and $p, r \in \beta T$ such that $t_{n} s_{n} \rightarrow p, t_{n} \rightarrow r$ and
(e) $x_{n} \rightarrow x_{1}$
(f) $x_{n}^{\prime} \rightarrow x_{1}^{\prime}$. 
Applying Lemma 1.3 to (b) and (e), and (d) and (f) we get that $x_{1} p=x=x_{1}^{\prime} p$. Since $\pi$ is distal, $x_{1}=x_{1}^{\prime}$. Applying Lemma 1.3 to (a) and (e), and (c) and (f) gives $x=x_{1} r$ and $x^{\prime}=x_{1}^{\prime} r$; hence $x=x^{\prime}$.

2. Highly proximal extensions. We temporarily drop the condition $X$ minimal; we still assume $Y$ minimal.

Proposition 2.1. The following are equivalent:

(i) All almost periodic elements of $2^{\pi}$ are singletons.

(ii) For some $y \in Y$ and net $\left\langle t_{n}\right\rangle$ in $T, \lim \left\langle\pi^{-1}(y) t_{n}\right\rangle$ is a singleton.

(iii) For some $y \in Y, p \in \beta T, \pi^{-1}(y) \circ p$ is a singleton.

Proof. (iii) $\Rightarrow$ (i). Suppose $A$ almost periodic in $2^{\pi}$ and card $\left(\pi^{-1}(y) \circ p\right)=1$. Pick $u \in J(y)$ and $y^{\prime} \in Y, u^{\prime} \in J, q \in M$ such that $A \circ u^{\prime}=A, A \subset \pi^{-1}\left(y^{\prime}\right)$, and $y p q=y^{\prime}$. Using Lemma 1.1,

$$
A \subset \pi^{-1}\left(y^{\prime}\right) \circ u^{\prime}=\pi^{-1}(y u p q) \circ u^{\prime}=\pi^{-1}(y) \circ\left(u p q u^{\prime}\right) \subset\left(\pi^{-1}(y) \circ p\right) \circ\left(q u^{\prime}\right),
$$

a singleton.

Definition. We call $\pi$ highly proximal if the conditions of 2.1 are satisfied.

Lemma 2.2. Consider a continuous map $\psi: A \rightarrow B$ where $A$ and $B$ are compact metric spaces. Then $\psi$ is open at all points of $\psi^{-1}(b)$ for a dense, $G_{\delta}$ set of points $b \in B$.

\section{Proof. See [6, 4.1.4].}

Proposition 2.3. If $X$ and $Y$ are metrizable, $\pi$ is highly proximal if and only if it is almost one-to-one.

Proof. $\Rightarrow$. By Lemma 2.2, there exists $y \in Y$ with $\pi$ open on $\pi^{-1}(y)$. If $u \in J(y)$, then $\pi^{-1}(y)=\pi^{-1}(y) \circ u$, a singleton.

Highly proximal is a nonmetric generalization of almost one-toone. It is a purely relative notion; it is easily seen that a highly proximal extension of the trivial flow is itself trivial. The Ellis two circle minimal flow [3, 5.29] taken as an extension of the circle with irrational rotation provides a nonmetric example of a homomorphism which is highly proximal but not almost one-to-one.

We establish some closure properties. A property of extensions of a fixed minimal flow is called admissible if it is nonvoid and closed under restriction of products to minimal sub-flows. 
Proposition 2.4. Highly proximal is admissible.

Proof. Suppose $\left\{\psi_{1}: W_{1} \rightarrow Y\right\}$ are highly proximal and $\psi: W \rightarrow Y$ is a restriction of their product to a minimal sub-flow. Pick $y \in Y$, $u \in J(y)$ and let $\psi_{i}^{-1}(y) \circ u=\left\{w_{i}\right\}$ for each $i$. Then $\psi^{-1}(y)=\left\{\left\langle w_{i}\right\rangle\right\}$.

LEMMA 2.5. Consider homomorphisms $\phi: U \rightarrow V, \psi: V \rightarrow W$, with all flows minimal. Then $\psi \circ \phi$ is highly proximal iff both $\phi$ and $\psi$ are.

Proof. Lemma 1.1.

LEMMA 2.6. Suppose $\left\{\phi_{1}: U_{1} \rightarrow V\right\}$ are proximal minimal flow homomorphisms with product $\phi: U \rightarrow V$. Then $U$ has a unique minimal subflow.

The category of point transitive flows with distinguished basepoints has unique inverse limits. Given an inverse system of minimal flows $\left\{U_{1}\right\}$ we can choose basepoints in a consistent way; we call the inverse limit of the point transitive system thus obtained a pointed inverse limit of $\left\{U_{1}\right\}$. We can always choose a minimal pointed inverse limit.

Proposition 2.7. Suppose we have an inverse system of minimal flows $\left\{U_{i}\right\}$ and highly proximal homomorphisms with a least element $U_{0}$. Then the system has a unique minimal pointed inverse limit $U_{\infty}$ and the canonical projections $P_{1}: U_{\infty} \rightarrow U_{1}$ are all highly proximal.

Proof. Proposition 2.4 and Lemmas 2.5 and 2.6.

Veech, in [7] associates with $\pi: X \rightarrow Y$ a diagram of the type

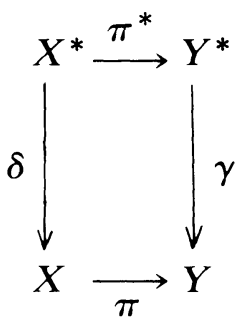

with $\pi^{*}$ open and $\delta$ and $\gamma$ almost one-to-one, assuming metrizability. We generalize by dropping metrizability and replacing almost one-to-one with highly proximal. Our construction will coincide with Veech's in the metric case.

Once more, we assume both $X$ and $Y$ minimal. We define 


$$
\begin{aligned}
& Y^{*}=\left\{\pi^{-1}(y) \circ u \mid y \in Y, u \in J(y)\right\} \quad \text { and } \\
& X^{*}=\left\{\left(x u, \pi^{-1}(y) \circ u\right) \mid y \in Y, x \in \pi^{-1}(y), u \in J(y)\right\} .
\end{aligned}
$$

We define $\gamma: Y^{*} \rightarrow Y$ by $\gamma(A)=y$ iff $A \subset \pi^{-1}(y)$. Finally, we define $\delta: X^{*} \rightarrow X$ and $\pi^{*}: X^{*} \rightarrow Y^{*}$ as the coordinate projections. It will be clear that these maps are all homomorphisms once we've shown that $X^{*}$ and $Y^{*}$ are minimal flows.

Proposition 2.8. With the definitions above

(i) $Y^{*}$ is the unique minimal subflow of $2^{\pi}$ contained in the orbit closure of some (every) $\pi$ fiber.

(ii) $X^{*}$ is the unique minimal set in $\left\{\left(x, y^{*}\right) \mid x \in X, y^{*} \in\right.$ $\left.Y^{*}, \pi(x)=\gamma(y)\right\}$.

Proof. (i) Consider minimal sets $M, M^{\prime}$ in $\beta T$ and points $y_{0}$, $y_{1} \in Y$. Lemma 1.1 and an enveloping semigroup argument show that $\pi^{-1}\left(y_{0}\right) \circ M=\pi^{-1}\left(y_{1}\right) \circ M^{\prime}$. This proves that there is a unique minimal subflow as asserted and that this subflow contains $Y^{*}$. Consider some $A=\pi^{-1}\left(y_{0}\right) \circ p$ in $\pi^{-1}\left(y_{0}\right) \circ M$. There exist $u, v \in J, y \in Y$ such that $y_{0} u=y, p v=p$, and $y=y_{0} p v$. Then $A=\pi^{-1}\left(y_{0} u\right) \circ(p v)=\pi^{-1}(y) \circ v$ by Lemma 1.1. Hence $Y^{*}=\pi^{-1}\left(y_{0}\right) \circ M$.

(ii) Consider $y \in Y, x \in \pi^{-1}(y), u \in J(y)$. A similar argument shows $X^{*}=\left(x u, \pi^{-1}(y) \circ u\right) M$.

Proposition 2.9. The homomorphisms $\gamma$ and $\delta$ are highly proximal.

Proof. Applying Zorn's Lemma to a fiber $\gamma^{-1}(y) \subset Y^{*}$ yields an inclusion minimal element $B$ with $B=\pi^{-1}(y) \circ p$ for some $p \in M$. Also $\gamma^{-1}(y) \circ p \subset \gamma^{-1}(y)$ and if $A \in \gamma^{-1}(y) \circ p$ then $A \subset B$. Hence $\gamma^{-1}(y) \circ p=$ $\{B\}$ and $\gamma$ is highly proximal. If $x \in X, u \in J(x)$ then $\delta^{-1}(x) \circ u \subset\{x\} \times$ $\gamma^{-1}(\pi(x)) \circ u$. Hence $\delta$ is also highly proximal.

For $y \in Y$, let $J M(y, \pi)$ be the set of idempotents $u \in J(y)$ such that $\pi^{-1}(y) \circ u$ is inclusion minimal in $Y^{*}$. Zorn's Lemma implies that $\operatorname{JM}(y, \pi)$ is always nonempty. The idea of the following lemma is due to Glasner.

Lemma 2.10. Suppose $u \in J M(y, \pi)$ and $x \in \pi^{-1}(y) \circ u$. Then there exists an idempotent $v \in J(x)$ such that $\pi^{-1}(y) \circ v=\pi^{-1}(y) \circ u$.

Proof. We can find nets $\left\langle x_{t}\right\rangle$ in $\pi^{-1}(y),\left\langle t_{t}\right\rangle$ in $T$, and $\left\langle p_{t}\right\rangle$ in $M$ such that $x p_{t}=x_{t}, t_{t} \rightarrow u, \quad x_{t} t_{t} \rightarrow x$, and $p_{t} t_{t} \rightarrow q$ for some $q \in M$. Then $\pi^{-1}(y) \circ\left(p_{i} t_{i}\right) \subset \pi^{-1}\left(y p_{i}\right) \circ t_{i}=\pi^{-1}(y) \circ t_{i}$, for each $i$, and so $\pi^{-1}(y) \circ q \subset$ 
$\pi^{-1}(y) \circ u$. Hence $\pi^{-1}(y) \circ q=\pi^{-1}(y) \circ u$ by minimality. Pick $v \in J$ such that $q v=q$. By Lemma 1.1, $\pi^{-1}(y) \circ v=\pi^{-1}(y q) \circ v=\pi^{-1}(y) \circ(q v)=$ $\pi^{-1}(y) \circ q=\pi^{-1}(y) \circ u$, and $x v=x q v=x q=x$.

Proposition 2.11. The homomorphism $\pi^{*}: X^{*} \rightarrow Y^{*}$ is open.

Proof. It suffices to show $\pi^{*-1}\left(y^{*} p\right) \subset \pi^{*-1}\left(y^{*}\right) \circ p$ for some $y^{*} \in$ $Y^{*}$ and any $p \in M$. Pick $y \in Y, u \in J M(y, \pi)$ and let $y^{*}=\pi^{-1}(y) \circ u$. Then $\pi^{*-1}\left(y^{*}\right)=\left(\pi^{-1}(y) \circ u\right) \times\left\{y^{*}\right\}$, by Lemma 2.10. It is easily seen that, for $p \in M$,

$$
\pi^{*-1}\left(y^{*} p\right) \subset\left(\pi^{-1}(y) \circ p\right) \times\left\{y^{*} p\right\}=\left(\left(\pi^{-1}(y) \circ u\right) \times\left\{y^{*}\right\}\right) \circ p=\pi^{*-1}\left(y^{*}\right) \circ p .
$$

We've shown that in the diagram

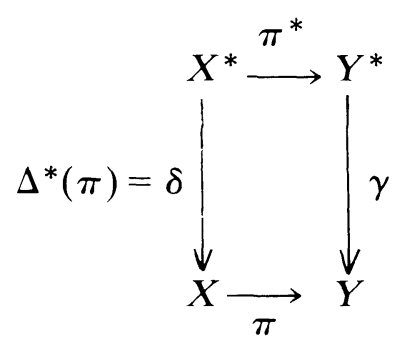

the homomorphisms $\delta$ and $\gamma$ are highly proximal while $\pi^{*}$ is open. We will use these properties to characterize $\Delta^{*}$ abstractly.

Lemma 2.12. We have $\pi$ open iff $\gamma$ (and hence also $\delta$ ) is an isomorphism.

Proof. $\Rightarrow$. For $y \in Y, \gamma^{-1}(y)=\left\{\pi^{-1}(y) \circ u \mid u \in J(y)\right\}=\left\{\pi^{-1}(y)\right\}$, since $\pi$ open.

$\Leftarrow$. We must show $\pi^{-1}(y p)=\pi^{-1}(y) \circ p$ for $y \in Y, p \in M$. Now $\gamma$ one-to-one implies $\gamma^{-1}(y p)=\left\{\pi^{-1}(y p)\right\}$ and $\pi^{-1}(y) \circ p \in \gamma^{-1}(y p)$ necessarily.

Clearly, if $\gamma$ is an isomorphism, so is $\delta$.

Lemma 2.13. Suppose $\beta$ is highly proximal in a diagram of minimal flows

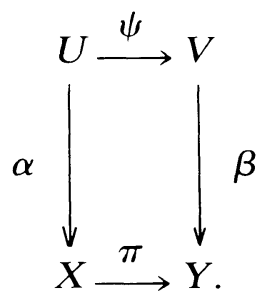


Then, for all $v \in V, \pi^{-1}(\beta(v))$ and $\alpha\left(\psi^{-1}(v)\right)$ are proximal in $2^{x}$.

Proof. We'll show $\left(\pi^{-1}(\beta(v))\right) \circ u=\left(\alpha\left(\psi^{-1}(v)\right)\right) \circ u$ for $u \in J(v)$. Clearly $\left(\alpha\left(\psi^{-1}(v)\right)\right) \circ u \subset\left(\pi^{-1}(\beta(v))\right) \circ u$. Now $\left(\alpha^{-1}\left(\pi^{-1}(\beta(v))\right)\right) \circ u=$ $\left(\psi^{-1}\left(\beta^{-1}(\beta(v))\right)\right) \circ u \subset \psi^{-1}\left(\beta^{-1}(\beta(v)) \circ u\right)=\psi^{-1}(v), \quad$ since $\quad \beta \quad$ highly proximal. Thus

$$
\begin{aligned}
\left(\pi^{-1}(\beta(v))\right) \circ u & =\left(\alpha\left(\alpha^{-1}\left(\pi^{-1}(\beta(v))\right)\right)\right) \circ u \\
& =\alpha\left(\alpha^{-1}\left(\pi^{-1}(\beta(v))\right) \circ u\right) \subset \alpha\left(\psi^{-1}(v)\right)
\end{aligned}
$$

and finally

$$
\left(\pi^{-1}(\beta(v))\right) \circ u=\left(\left(\pi^{-1}(\beta(v))\right) \circ u\right) \circ u \subset\left(\alpha\left(\psi^{-1}(v)\right)\right) \circ u .
$$

Given minimal flow diagrams
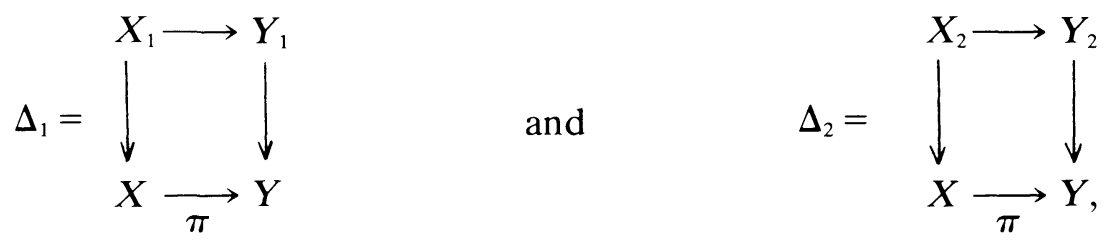

we say that $\Delta_{1}$ is less than $\Delta_{2}$ if there exist homomorphisms from $X_{2}$ to $X_{1}$ and from $Y_{2}$ to $Y_{1}$ making everything commute.

THEOREM 2.14. The diagram $\Delta^{*}(\pi)$ is the unique (up to isomorphism) least such diagram with $\pi^{*}$ open and $\gamma$ highly proximal.

Proof. Consider another such diagram

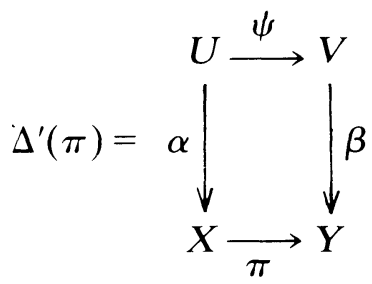

with $\psi$ open and $\beta$ highly proximal. We apply the $\Delta^{*}$ construction to both $\pi$ and $\psi$ to obtain 


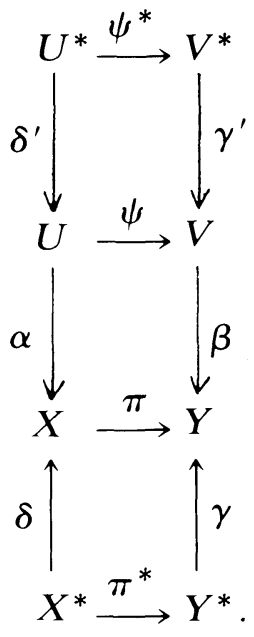

We'll show that there exist homomorphisms $\alpha^{\prime}: U^{*} \rightarrow X^{*}$, $\beta^{\prime}: V^{*} \rightarrow Y^{*}$ making everything commute. Pick $v \in V$. By Lemma 2.13 , there exists a minimal right ideal $I$ in $\beta T$ such that $\left(\pi^{-1}(\beta(v))\right) \circ p=$ $\left(\alpha\left(\psi^{-1}(v)\right)\right) \circ p$ for all $p \in I$. Pick an idempotent $u \in I$ such that $v u=v$, let $y=\beta(v), v^{*}=\psi^{-1}(v)^{\circ} u$, and $y^{*}=\pi^{-1}(y) \circ u$. To obtain $\beta^{\prime}$ with $\beta^{\prime}\left(v^{*}\right)=y^{*}$ it will suffice to show that for $p, q \in \beta T$, if $v^{*} p=v^{*} q$ then $y^{*} p=y^{*} q$. Now

$$
\begin{aligned}
y^{*} p & =\left(\pi^{-1}(y) \circ u\right) \circ p=\left(\pi^{-1}(\beta(v))\right) \circ u \circ p=\left(\alpha\left(\psi^{-1}(v)\right)\right) \circ u \circ p \\
& =\alpha\left(v^{*} p\right)=\alpha\left(v^{*} q\right)=y^{*} q .
\end{aligned}
$$

We obtain $\alpha^{\prime}$ by a similar argument.

Since $\psi$ is open, $\delta^{\prime}$ and $\gamma^{\prime}$ are actually isomorphisms, by Lemma 2.12. Thus $\Delta^{*}$ is less than $\Delta^{\prime}$. To prove uniqueness consider another such diagram

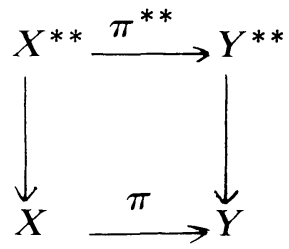

with the same minimality property. Then we have homomorphisms $\lambda: X^{* *} \rightarrow X^{*}, \quad \lambda^{\prime}: X^{*} \rightarrow X^{* *}$ such that $\delta^{* *} \circ \lambda^{\prime}=\delta$ and $\delta \circ \lambda=$ $\delta^{* *}$. We have $\delta \circ\left(\lambda \circ \lambda^{\prime}\right)=\delta$ and $\delta$ is a proximal homomorphism; it follows that $\lambda \circ \lambda^{\prime}$ is an automorphism and hence $\lambda^{\prime}$ an 
isomorphism. The same argument provides the required isomorphism from $Y^{*}$ to $Y^{* *}$.

The homomorphism $\pi: X \rightarrow Y$ is said to be point-distal with distal point $x \in X$ if $x$ is proximal to no other point in its fiber. The minimal flow $X$ is point-distal if the trivial homomorphism $X \rightarrow 1$ has that property. Veech [7] showed that if $X$ is metric and has a residual set of distal points it has an almost one-to-one extension which can be built up from the trivial flow by isometric (almost periodic) and almost one-to-one extensions. Ellis [4] extended this result to homomorphisms and showed that it is sufficient to assume a single distal point rather than a residual set. He also showed that the metrizability assumption could be replaced by the weaker condition of quasi-separability if proximal extensions were used instead of almost one-to-one extensions. Here we strengthen the second Ellis result by replacing his proximal extensions by highly proximal extensions. Since highly proximal and almost one-toone extensions are the same in the metric case, this result includes the earlier ones.

The homomorphism $\Pi: X \rightarrow Y$ is said to be quasi-separable if it is isomorphic to a restriction of the projection onto $Y$ of the product of $Y$ and a family of metrizable flows. For the rest of this section we assume both $X$ and $Y$ minimal and $\pi$ point-distal and quasi-separable. Under these hypotheses Ellis [4, 7.4] showed

LEMMA 2.15. If $\pi$ is also open there exists a nontrivial almost periodic homomorphism $\phi: Z \rightarrow Y$ and a homomorphism $\psi: X \rightarrow Z$ such that $\pi=\phi \circ \psi$. In other words, $\pi$ has a nontrivial almost periodic factor.

THEOREM 2.16. There exists an ordinal sequence of minimal sets $\left\{Y_{\alpha} \mid \alpha \leqq \nu\right\}$ such that

(i) $\quad Y_{0}=Y$.

(ii) $Y_{\nu}$ is a highly proximal extension of $X$.

(iii) $Y_{\alpha+1}$ is either an almost periodic or a highly proximal extension of $Y_{\alpha}$, for each successor ordinal $\alpha+1 \leqq \nu$.

(iv) $Y_{\lambda}$ is a uniquely determined pointed inverse limit of the system $\left\{Y_{\alpha} \mid \alpha<\lambda\right\}$ for each limit ordinal $\lambda \leqq \nu$.

(v) If $\eta_{\nu}: Y_{\nu} \rightarrow X$, and $\mu_{\alpha, \beta}: Y_{\alpha} \rightarrow Y_{\beta}$ for ordinals $\alpha<\beta$ are the homomorphisms implicitly defined by (i)-(iv), then $\pi \circ \eta_{\nu}=\mu_{\nu, 0}$.

Proof. The proof is by transfinite induction. At each ordinal stage $\beta \leqq \nu$ we'll get a diagram 


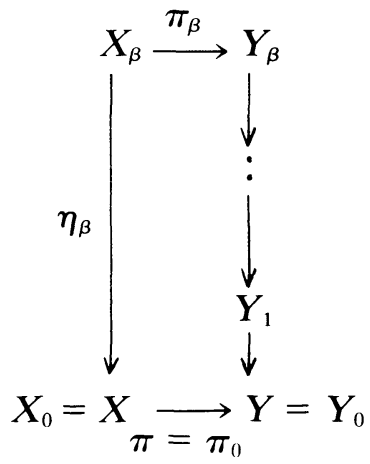

such that $Y_{\beta}$ is as promised, $\eta_{\beta}$ is highly proximal, and $\pi_{\beta}$ is point-distal and quasi-separable. The procedure stops when $\pi_{b}$ is almost periodic. Call an ordinal odd if it's of the form $\lambda+n$ where $\lambda$ is a limit ordinal or 0 , and $n$ is an odd natural number. Proceed as follows.

(a) Take $X_{0}=X, Y_{0}=Y$, and $\pi_{0}=\pi$.

(b) At stage $\alpha+1$, with $\alpha+1$ an odd successor ordinal, stop if $\pi_{\alpha}$ is almost periodic, taking $\nu=\alpha+1$ and $Y_{\nu}=X_{\alpha}$. Otherwise, construct the diagram

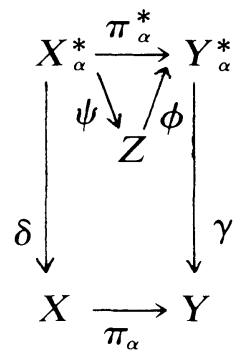

as follows. Obtain $\pi_{\alpha}^{*}$ as usual. Then $\pi_{\alpha}^{*}$ is open, $\gamma$ and $\delta$ are highly proximal, and $\pi_{\alpha}^{*}$ is easily seen to be point-distal and quasiseparable. By Lemma $2.15, \pi_{\alpha}^{*}$ has a nontrivial almost periodic factor, $\phi: Z \rightarrow Y_{\alpha}^{*}$. Clearly since $\pi_{\alpha}^{*}$ is point-distal and quasi-separable, so is $\psi: X_{\alpha}^{*} \rightarrow Z$. Take $Y_{\alpha+1}=Y_{\alpha}^{*}, \quad Y_{\alpha+2}=Z, X_{\alpha+1}=X_{\alpha+2}=X_{\alpha}^{*}, \pi_{\alpha+1}=\pi_{\alpha}^{*}$, and $\pi_{\alpha+2}=\psi$. We now have

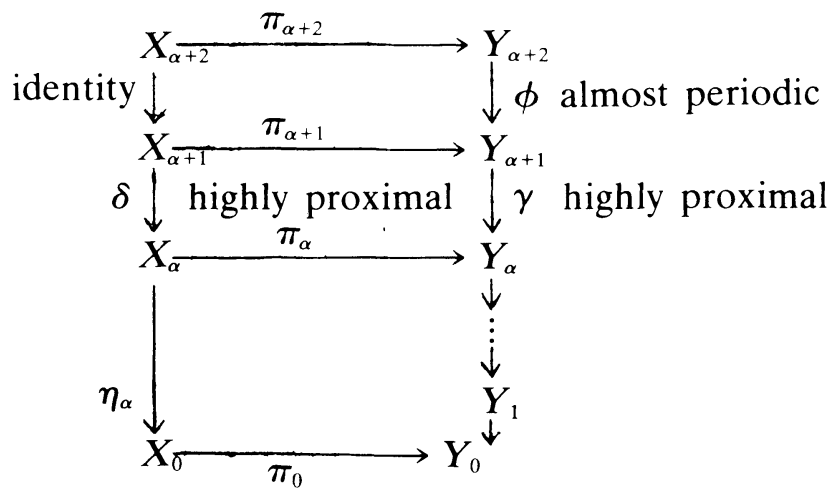


and everything is as needed, taking $\eta_{\alpha+1}=\eta_{\alpha} \circ \delta$. This gets us up to the next odd successor ordinal.

(c) At stage $\lambda$, where $\lambda$ is a limit ordinal, let $X_{\lambda}$ be the unique (by Proposition 2.7) pointed inverse limit of the system $\left\{X_{\alpha} \mid \alpha<\lambda\right\}$ and let $P_{\alpha}: X_{\lambda} \rightarrow X_{\alpha}$ be the projections which are highly proximal. Define $Q: \Pi\left\{X_{\alpha} \mid \alpha<\lambda\right\} \rightarrow \Pi\left\{Y_{\alpha} \mid \alpha<\lambda\right\}$ by $Q\left(\left\langle x_{\alpha}\right\rangle\right)=\left\langle\pi_{\alpha} \circ P_{\alpha}\left(x_{\alpha}\right)\right\rangle$. Let $Y_{\lambda}=$ $Q\left(X_{\lambda}\right), \pi_{\lambda}=Q \mid X_{\lambda}$, and $\eta_{\lambda}=P_{0} \mid X_{\lambda}$. Then $\pi_{\lambda}$ is point-distal and quasiseparable and everything is as needed.

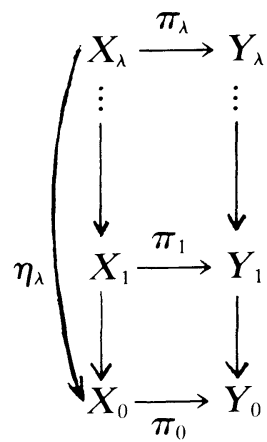

The definition of the maps $\mu_{\alpha, \beta}: Y_{\alpha} \rightarrow Y_{\beta}$ has been left to context. By cardinality considerations and the coalescence of the universal minimal set, the procedure must terminate at some stage of (b). This proves the theorem.

3. Generalized almost finite extensions. We assume minimality of $Y$ but not of $X$.

Lemma 3.1. Suppose $A \in 2^{x}, p \in \beta T$. If $A$ is finite, then $A p=$ $A \circ p$.

Proof. $A p \subset A \circ p$ in any case. For $x \in A \circ p$, there exist nets $\left\langle x_{m}\right\rangle$ in $A,\left\langle t_{m}\right\rangle$ in $T$ such that $t_{m} \rightarrow p$ and $x_{m} t_{m} \rightarrow x$. Since $A$ is finite, $\left\langle x_{m}\right\rangle$ has a constant subnet. Hence for a subnet and some $x^{\prime} \in A, x^{\prime} t_{m} \rightarrow x$. Thus $x=x^{\prime} p$ and $x \in A p$.

Proposition 3.2. The following conditions are equivalent:

(i) $\operatorname{Card}\left(\pi^{-1}(y) \circ p\right)=N$ for some $y \in Y, p \in M$, and integer $N$.

(ii) The cardinality of each almost periodic element in $2^{\pi}$ is not greater than some fixed integer $N$.

(iii) All elements of $Y^{*}$ have the same finite cardinality, $N$.

Moreover, the integer $N$ in (iii) equals the least integer satisfying (i) and (ii). 
Proof. Use Lemmas 1.1, 3.1 and the minimality of $Y$.

DEFINITION. We call $\pi$ generalized almost finite $(G A F)$, or generalized almost $N$ to one if the conditions of Proposition 3.2 are satisfied.

LEMma 3.3. If $\pi$ is $G A F, y \in Y$, and $u \in J(y)$, then $\pi^{-1}(y) \circ u=$ $\pi^{-1}(y) u$.

Proposition 3.4. If $\pi$ is generalized almost $N$ to one the following are equivalent, for any $y \in Y$.

(i) $\pi$ is open on $\pi^{-1}(y)$.

(ii) $\operatorname{Card}\left(\pi^{-1}(y)\right)=N$.

(iii) $\pi^{-1}(y)$ is an almost periodic set.

(iv) $\pi^{-1}(y) \in Y^{*}$.

Proof. Use Lemma 2.3 to prove (iii) $\Leftrightarrow$ (iv). The rest is straightforward.

Proposition 3.5. If $X$ is metric the following are equivalent:

(i) $\pi$ is $G A F$.

(ii) Some fiber is finite.

(iii) $Y$ has a dense, $G_{\delta}$ subset of points with finite fibers.

Proof. Lemma 2.2 and Propositions 3.2 and 3.4.

We say that a minimal flow homomorphism is regular $[6,2]$ if every almost periodic pair of points contained in a common fiber is connected by an automorphism. If every pair contained in a common fiber is so connected we say we have a group extension. We now assume that $X$ is minimal as well as $Y$.

There are nonmetric examples of homomorphisms which are GAF (in fact highly proximal) but which have all fibers infinite. Suppose $\pi: X \rightarrow Y$ is almost one-to-one but has some infinite fiber and $Y$ is distal regular, so that every pair in $Y$ is connected by an automorphism. Let $\phi: N \rightarrow Y$ be the restriction of the product $\Pi\{\theta \circ \pi \mid \theta \in$ Aut $(Y)\}$ to a minimal subflow. Then $\phi$ will be highly proximal but will have all fibers infinite. The Floyd minimal flow [1], taken as an extension of the triadic group provides an example of $\pi$ as required.

Next we characterize regular, GAF homomorphisms. Recall the natural map $\gamma: Y^{*} \rightarrow Y$.

Proposition 3.6. If $\pi$ is regular and GAF there exists a group extension $\psi: X \rightarrow Y^{*}$ such that $\pi=\gamma \circ \psi$. 
Proof. It follows from the regularity of $\pi$ and Lemma 3.3 that the fibers of $\pi$ are partitioned by the elements of $Y^{*}$. Thus we define $\psi: X \rightarrow Y^{*}$ by $\psi(x)=A \Leftrightarrow x \in A$. It is easily seen that $\psi$ is a group extension and $\pi=\gamma \circ \psi$.

Proposition 3.7. The following are equivalent:

(i) $\pi$ is regular and GAF.

(ii) There exists a finite group extension $\phi$ and a highly proximal homomorphism $\alpha$ such that $\pi=\alpha \circ \phi$.

Moreover, the representation in (ii) is unique.

Proof. (ii) $\Rightarrow$ (i). Suppose $\left(x, x^{\prime}\right)$ is an almost periodic pair with $\pi(x)=\pi\left(x^{\prime}\right)=y$. Pick $u \in J(y)$ such that $\left(x, x^{\prime}\right) u=\left(x, x^{\prime}\right)$. Then $\phi(x)=\phi\left(x^{\prime}\right)$, since $\phi(x), \phi\left(x^{\prime}\right) \in \alpha^{-1}(y) \circ u$, a singleton. Thus we get an automorphism taking $x$ into $x^{\prime}$, so $\pi$ is regular. Also $\pi^{-1}(y) \circ u \subset$ $\phi^{-1}\left(\alpha^{-1}(y) \circ u\right)$ which is finite. Thus $\pi$ is GAF.

(i) $\Rightarrow$ (ii) has already been shown; the uniqueness follows easily.

Many examples of regular GAF extensions may be constructed by taking products of highly proximal and finite group extensions or by taking finite group extensions of highly proximal extensions. The following lemma is proved in [6].

LEMMA 3.8. The relative (to $\pi$ ) proximal relation on $X$ is closed iff $\pi=\beta \circ \alpha$ for some proximal $\alpha$ and distal $\beta$.

Proposition 3.9. Suppose $\pi_{1}: X_{1} \rightarrow Y, \pi_{2}: X_{2} \rightarrow Y$ are highly proximal and finite group extensions respectively with product $\pi: X \rightarrow Y$. Then:

(i) $\pi$ is regular $G A F$.

(ii) $\pi$ is a highly proximal extension of a group extension.

(iii) The automorphism groups of the two group extensions are isomorphic.

Proof. We have the diagram

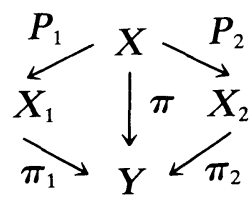

where $P_{1}$ and $P_{2}$ are the projections. $X$ is minimal, since proximal and distal extensions are always disjoint. Suppose $x_{2} \in X_{2}, u \in J\left(x_{2}\right)$. Let $\left\{x_{1}\right\}=\pi_{1}^{-1}\left(\pi_{2}\left(x_{2}\right)\right) \circ u$. Then clearly $P_{2}^{-1}\left(x_{2}\right) \circ u=\left\{\left(x_{1}, x_{2}\right)\right\}$, so $P_{2}$ is 
highly proximal. Let Aut $\left(\pi_{2}\right)=\left\{\theta \in\right.$ Aut $\left.\left(X_{2}\right) \mid \pi_{2} \circ \theta=\pi_{2}\right\}$ and Aut $\left(P_{1}\right)=\left\{\hat{\theta} \in \operatorname{Aut}(X) \mid P_{1} \circ \hat{\theta}=P_{1}\right\}$. Define $F$ : Aut $\left(\pi_{2}\right) \rightarrow \operatorname{Aut}\left(P_{1}\right)$ by $F(\theta)\left(x_{1}, x_{2}\right)=\left(x_{1}, \theta\left(x_{2}\right)\right)$. It is clear that $F$ is a group isomorphism and that $P_{1}$ is a group extension.

THEOREM 3.10. If $\pi$ is regular, GAF the following are equivalent:

(i) The relative proximal relation on $X$ is closed.

(ii) $\pi=\beta \circ \alpha$ for some highly proximal $\alpha$ and finite group extension $\beta$.

(iii) $\pi$ is the product of a highly proximal and a finite group extension.

Proof. (iii) $\Rightarrow$ (ii) $\Rightarrow$ (i). Proposition 3.9 and Lemma 3.8.

(i) $\Rightarrow$ (ii). By 3.6 and 3.8 we have a diagram

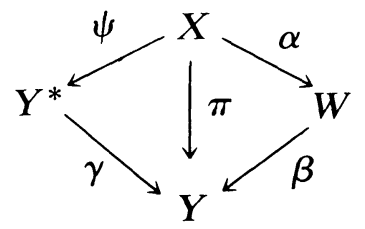

with $\gamma$ highly proximal, $\psi$ a finite group extension, $\beta$ distal, and $\alpha$ proximal. Also, $\alpha$ is $G A F$, since $\pi$ is; hence $\alpha$ is in fact highly proximal. Suppose $y \in Y, u \in J(y)$. Since $\alpha$ is proximal and $\beta$ distal, $\alpha$ maps $\pi^{-1}(y) u$ bijectively onto $\beta^{-1}(y)$; hence $\beta$ has finite fibers. It now suffices to show that for arbitrary $w_{1}, w_{2} \in \beta^{-1}(y)$, we can define an endomorphism $\hat{\theta}$ by $\hat{\theta}\left(w_{1}\right)=\hat{\theta}\left(w_{1}\right)=w_{2}$. Since $\gamma$ is proximal and $\psi$ a group extension, it follows that for any almost periodic pair $\left(x_{1}, x_{2}\right)$ with $\alpha\left(x_{l}\right)=w_{l}$, there exists $\theta \in \operatorname{Aut}(X)$ with $\theta\left(x_{1}\right)=x_{2}$. From this the existence of $\hat{\theta}$ follows easily.

(ii) $\Rightarrow$ (iii). We get a diagram

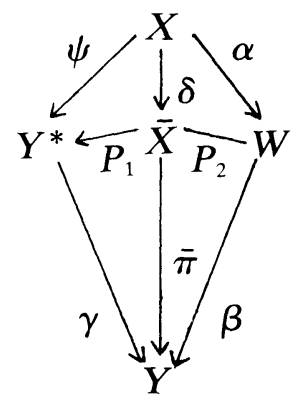

where $\bar{\pi}: \bar{X} \rightarrow Y$ is the product of $\gamma$ and $\beta, P_{1}$ and $P_{2}$ are the projections, $\delta$ is defined naturally, and $\pi=\bar{\pi} \circ \delta=\beta \circ \alpha=\gamma \circ \psi$. We need only 
show that $\delta$ is one-to-one. Now if $\delta(x)=\delta\left(x^{\prime}\right)$, then $\psi(x)=\psi\left(x^{\prime}\right)$ and $\alpha(x)=\alpha\left(x^{\prime}\right)$. We have $\psi$ distal and $\alpha$ proximal, so the pair $\left(x, x^{\prime}\right)$ is both proximal and almost periodic. Thus $x=x^{\prime}$.

We close with the following result.

Proposition 3.11. If $\pi$ is GAF, then $\pi^{*}$ is almost periodic.

Proof. Suppose $y^{*} \in Y^{*}$ and $A \subset \pi^{*-1}\left(y^{*}\right)$. By 1.4, we need only show $A \circ u=A$ for some $u \in J$. Now $y^{*}=\pi^{-1}(y) \circ u$ for some $y \in Y$, $u \in J(y)$. Since $\pi$ is GAF, $\pi^{*-1}\left(y^{*}\right)=\pi^{-1}(y) u \times\left\{y^{*}\right\}$, a finite set. Thus $A \circ u=A u=A$, by 3.1 .

An interesting question is whether the results of this section can be obtained with a hypothesis weaker than GAF, not involving any finiteness assumption. This will be the subject of a subsequent paper.

\section{REFERENCES}

1. J. Auslander, Mean L-stable systems, Illinois J. Math., 3 (1959), 566-579.

2. — Regular minimal sets, I, Trans. Amer. Math. Soc., 123 (1966), 469-479.

3. R. Ellis, Lectures on Topological Dynamics, W. A. Benjamin, New York, 1969.

4. — The Veech structure theorem, Trans. Amer. Math. Soc., 186 (1973), 203-218.

5. S. Glasner, Compressibility properties in topological dynamics, Amer. J. Math., 97 (1975), 148-171.

6. P. Shoenfeld, Regular Homomorphisms of Minimal Sets, Doctoral Dissertation, University of Maryland, 1974.

7. W. Veech, Point distal flows, Amer. J. Math., 92 (1970), 205-242.

Received March 21, 1975 and in revised form March 22, 1976. These results are taken from a dissertation [6] written under the direction of Joseph Auslander. The author wishes to thank Professor Auslander for his advice, encouragement, and suggestions. 



\section{Pacific Journal of Mathematics}

\section{Vol. 66, No. $1 \quad$ November, 1976}

Helen Elizabeth. Adams, Factorization-prime ideals in integral domains ............ Patrick Robert Ahern and Robert Bruce Schneider, The boundary behavior of Henkin's kernel.

Daniel D. Anderson, Jacob R. Matijevic and Warren Douglas Nichols, The Krull

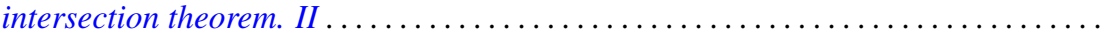

Efraim Pacillas Armendariz, On semiprime P.I.-algebras over commutative regular

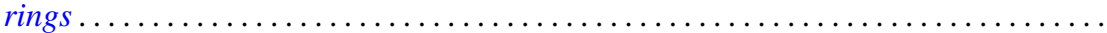

Robert H. Bird and Charles John Parry, Integral bases for bicyclic biquadratic fields

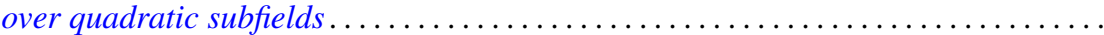

Tae Ho Choe and Young Hee Hong, Extensions of completely regular ordered

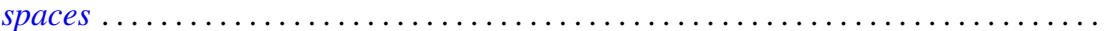

John Dauns, Generalized monoform and quasi injective modules ...............

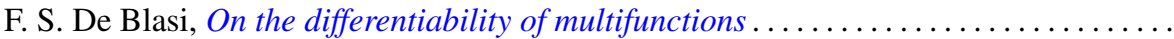

Paul M. Eakin, Jr. and Avinash Madhav Sathaye, R-endomorphisms of $R[[X]]$ are

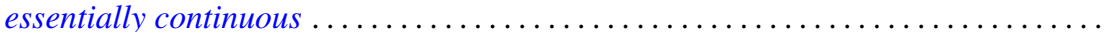

Larry Quin Eifler, Open mapping theorems for probability measures on metric

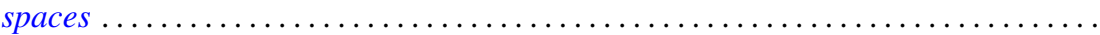

Garret J. Etgen and James Pawlowski, Oscillation criteria for second order self adjoint

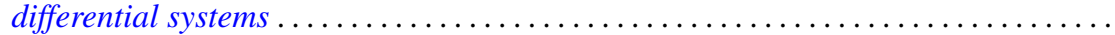

Ronald Fintushel, Local $S^{1}$ actions on 3-manifolds .

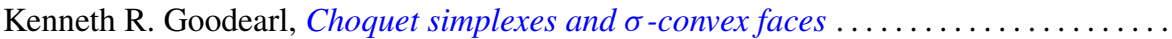

John R. Graef, Some nonoscillation criteria for higher order nonlinear differential

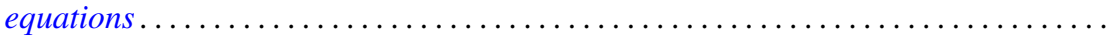

Charles Henry Heiberg, Norms of powers of absolutely convergent Fourier series: an example.

Les Andrew Karlovitz, Existence of fixed points of nonexpansive mappings in a space

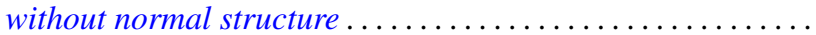

Gangaram S. Ladde, Systems of functional differential inequalities and functional

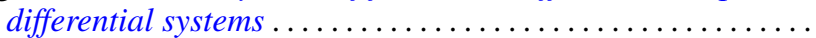

Joseph Michael Lambert, Conditions for simultaneous approximation and interpolation with norm preservation in $C[a, b]$.

Ernest Paul Lane, Insertion of a continuous function.

Robert F. Lax, Weierstrass points of products of Riemann surfaces .

Dan McCord, An estimate of the Nielsen number and an example concerning the Lefschetz fixed point theorem...

Paul Milnes and John Sydney Pym, Counterexample in the theory of continuous functions on topological groups...

Peter Johanna I. M. De Paepe, Homomorphism spaces of algebras of holomorphic functions

Judith Ann Palagallo, A representation of additive functionals on $L^{p}$-spaces,

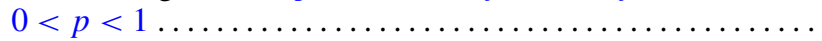

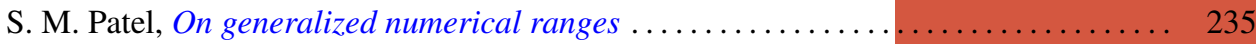

Thomas Thornton Read, A limit-point criterion for expressions with oscillatory

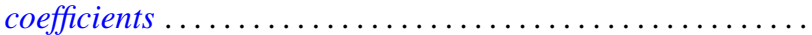

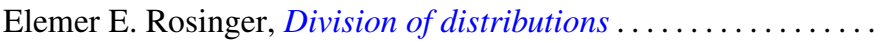

Peter S. Shoenfeld, Highly proximal and generalized almost finite

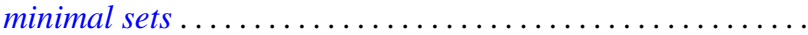

R. Sirois-Dumais and Stephen Willard, Quotient-universal sequential spaces

Robert Charles Thompson, Convex and concave functions of singular values of matrix sums....

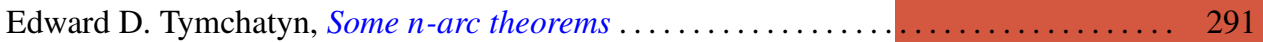

